\title{
Hydrodynamic Boundary Conditions and Dynamic Forces between Bubbles and Surfaces
}

\author{
Ofer Manor, ${ }^{1}$ Ivan U. Vakarelski, ${ }^{1}$ Xiaosong Tang, ${ }^{2}$ Sean J. O’Shea, ${ }^{2}$ Geoffrey W. Stevens, ${ }^{1}$ Franz Grieser, ${ }^{1}$ \\ Raymond R. Dagastine, ${ }^{1, *}$ and Derek Y. C. Chan ${ }^{1,3,4, \dagger}$ \\ ${ }^{1}$ Particulate Fluids Processing Centre, University of Melbourne, Parkville, Victoria 3010, Australia \\ ${ }^{2}$ Institute of Materials Research and Engineering, 3 Research Link, 117602, Singapore \\ ${ }^{3}$ Department of Mathematics, National University of Singapore, 117543, Singapore \\ ${ }^{4}$ Institute of High Performance Computing, 1 Science Park Road, 117528, Singapore
}

(Received 9 April 2008; published 11 July 2008)

\begin{abstract}
Dynamic forces between a $50 \mu \mathrm{m}$ radius bubble driven towards and from a mica plate using an atomic force microscope in electrolyte and in surfactant exhibit different hydrodynamic boundary conditions at the bubble surface. In added surfactant, the forces are consistent with the no-slip boundary condition at the mica and bubble surfaces. With no surfactant, a new boundary condition that accounts for the transport of trace surface impurities explains variations of dynamic forces at different speeds and provides a direct connection between dynamic forces and surface transport effects at the air-water interface.
\end{abstract}

DOI: 10.1103/PhysRevLett.101.024501

PACS numbers: 47.61.Jd, 47.55.dd, 47.55.nb

Hydrodynamic boundary conditions and dynamic forces involving deformable bodies on the nanometer length scale underpin developments in micro- and nanofluidic engineering applications [1] and in biomedical modeling [2]. For example, micrometer-sized bubbles are used in ultrasonic diagnostic applications and as drug delivery vectors [3]. We report atomic force microscope (AFM) measurements of dynamic forces between a bubble $(\sim 50 \mu \mathrm{m}$ radius) anchored at the end of a custom-made cantilever that is driven towards or separated from a molecularly smooth mica plate in a simple electrolyte and in the presence of surfactants. Hydrodynamic interactions and micabubble electrical double layer repulsion across aqueous films down to $\sim 30 \mathrm{~nm}$ thick cause the bubble to deform. We can determine the hydrodynamic boundary condition that must hold at the air-water interface, the simplest smooth and deformable interface whose interfacial properties can be controlled by the addition of surfactants, by modeling the dynamic force. In the presence of the surfactant sodium dodecylsulfate (SDS) above its critical micelle concentration, measured forces over a range of drive speeds are in excellent agreement with a model of bubble deformations based on the Young-Laplace equation and hydrodynamic interactions described by the Reynolds lubrication theory with no-slip boundary conditions at the bubble and mica surfaces. However, in electrolyte but no surfactants, experimental results lie between that predicted by the no-slip boundary condition (a fully immobile interface) and the full-slip boundary condition (continuity of tangential stress across a fully mobile interface) at the bubble surface. A model that allows for dynamic transport of trace adsorbed surface impurities at the air-water interface during interaction is able to account for the observations. This study provides the first direct connection between dynamic forces and surface species at molecularly smooth and deformable interfaces in the limit of negligible viscosity ratio between the interior and the surrounding phase.

The no-slip boundary condition is known to be consistent with measured hydrodynamic forces between two molecularly smooth mica sheets across wetting liquids down to subnanometer separations [4-8] and with direct observations [9,10] and modeling [11-13] of drainage and deformations of subnanometer thick aqueous films between a deformable mercury drop and a moving mica plate. However, a recent study [14] reported Navier slip lengths (ratio of fluid velocity to the tangential velocity gradient at the surface) of up to $20 \mathrm{~nm}$ using a similar apparatus although the deduced slip length can vary by an order of magnitude depending on the method of data analysis. Dynamic force measurements using the AFM with micrometer-sized particles reported evidence of hydrodynamic slip at wetted surfaces [15-19] although a recent AFM study concluded that the slip length in such systems is zero within experimental uncertainty of $\pm 1 \mathrm{~nm}$ [20]. AFM measurements of hydrodynamic forces between deformable oil drops stabilized by surfactants in water [21-25], between such drops and solid particles [26], and in a recent study of the dynamic response of a film of glycerol-water mixture between a millimeter particle and a bubble raft [27], as well as the dynamic drainage of micrometer thick silicone oil films between approaching millimeter size glycerol drops [28], all indicate a no-slip hydrodynamic boundary condition at the fluid interfaces.

We used ultrasound to generate large populations of bubbles from several tens to several hundred micrometers in size on weakly hydrophobic surfaces [29]. A bubble of $\sim 50 \mu \mathrm{m}$ radius was then picked up and anchored to a hydrophobized circular gold patch of $40 \mu \mathrm{m}$ diameter (thickness $20 \mathrm{~nm}$ ) that was centered at $5 \mu \mathrm{m}$ from the end of a custom-manufactured rectangular silicon AFM cantilever of dimensions: $450 \mu \mathrm{m} \times 50 \mu \mathrm{m} \times 3 \mu \mathrm{m}$ 
(Fig. 3). The spring constant of the cantilever $K(90 \pm$ $10 \mathrm{mN} / \mathrm{m}$ ) was determined by the Cleveland method [30]. The dynamic force between such a bubble and a freshly cleaved mica plate was first measured in distilled water with $1 \mathrm{mM} \mathrm{NaNO}{ }_{3}$ electrolyte. With the bubble remaining on the cantilever, the electrolyte was exchanged for a $10 \mathrm{mM}$ SDS solution to yield a monolayer of SDS adsorbed at the bubble surface. The dynamic force between this surfactant coated bubble and the mica is then measured in the presence of the SDS solution. SDS did not adsorb significantly onto mica as it was negatively charged. The bubble size was monitored at the beginning and end of each force run to confirm that it did not change significantly although the bubbles shrank in size by about $10 \%$ due to slow gas dissolution during the 20-30 min experiment in electrolyte and in SDS solution.

The experiments were carried out on an Asylum MFP3D AFM equipped with a linear variable differential transformer (LVDT) which reports the actual location $X(t)$ (which is not linear in time) of the $z$-piezo in the approach or retract cycle of a force-displacement measurement. The measured forces $F(t)$ in the presence of SDS are shown in Fig. 1 as functions of time and of the LVDT displacement $X(t)$. The force $F(t)$ can be modeled by an axi-symmetric hydrodynamic model (Fig. 2) that couples the thickness $h(r, t)$ of the water film between the mica and the deform-

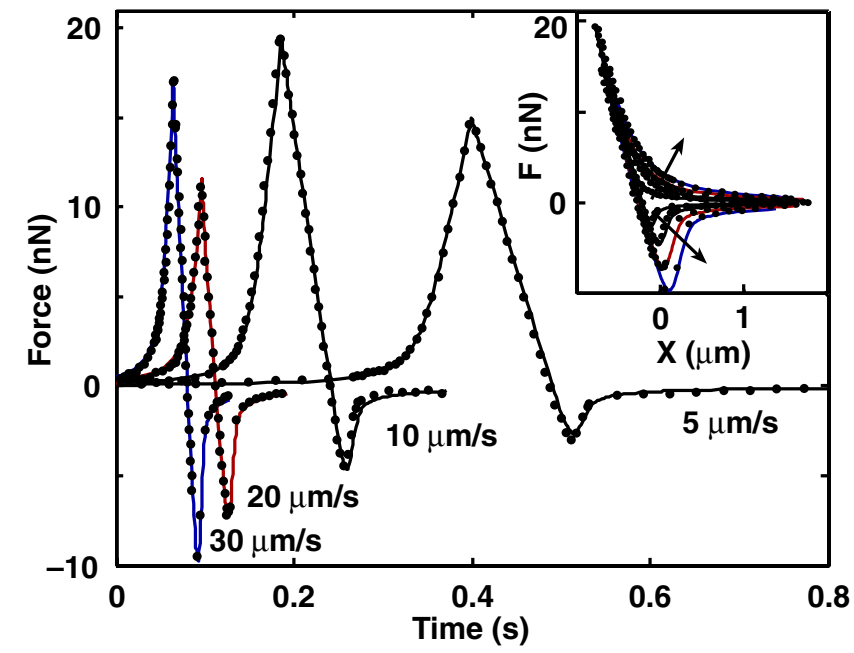

FIG. 1 (color online). Measured dynamic forces $F(t)$ between a bubble and a mica plate in $10 \mathrm{mM}$ SDS at indicated AFM scan rates (dotted line) and the model with the no-slip boundary condition at the bubble and mica surfaces (solid line). Model parameters with experimental values in brackets: mica surface potential: $-70 \mathrm{mV}(-75 \pm 10 \mathrm{mV}$ [34]), bubble surface potential: $-65 \mathrm{mV}(-60 \pm 10 \mathrm{mV}$ [35]), interfacial tension $\sigma$ : $39 \mathrm{mN} / \mathrm{m}(39 \pm 1 \mathrm{mN} / \mathrm{m})$, bubble radius $R_{b}: 47 \mu \mathrm{m}(50 \pm$ $3 \mu \mathrm{m})$ and contact angle $\theta: 142^{\circ}\left(147 \pm 5^{\circ}\right)$. The initial separations $h_{\text {init }}$ are: $2.05 \mu \mathrm{m}(5 \mu \mathrm{m} / \mathrm{s}), 1.80 \mu \mathrm{m}(10 \mu \mathrm{m} / \mathrm{s})$, $1.98 \mu \mathrm{m}(20 \mu \mathrm{m} / \mathrm{s})$ and $1.87 \mu \mathrm{m}(30 \mu \mathrm{m} / \mathrm{s})$. Inset: $F(t)$ against cantilever displacement $X(t)$, where arrows indicate the directions of increasing scan rate. ing bubble, the hydrodynamic pressure $p(r, t)$ and the disjoining pressure $\Pi(h(r, t))$ due in the present system to electrical double layer interactions calculated using the nonlinear Poisson-Boltzmann theory [31]. van der Waals interactions are negligible in the range of film thicknesses in the present system. With a constant air-water interfacial tension $\sigma$ and a Newtonian viscosity $\mu\left(10^{-3} \mathrm{Pas}\right)$ for the aqueous film, the governing equations are $[13,21]$

$$
\begin{gathered}
\frac{\sigma}{r} \frac{\partial}{\partial r}\left[r \frac{\partial h}{\partial r}\right]=\Delta P-(p+\Pi), \\
\frac{\partial h}{\partial t}=-\frac{1}{r} \frac{\partial}{\partial r}[r Q] \text { and } \mu \frac{\partial^{2} u}{\partial z^{2}}=\frac{\partial p}{\partial r} .
\end{gathered}
$$

Equation (1) is the augmented Young-Laplace equation that relates variations of the aqueous film thickness $h$ to hydrodynamic and disjoining pressure variations across the film. The thinning or thickening of the aqueous film as a bubble approaches or retracts from the mica is governed by Eq. (2). These follow from the Reynolds lubrication theory for the dominant radial component of the fluid velocity $u(z, t)$ with $Q(r, t) \equiv \int_{0}^{h} u(z, t) d z$. The time-dependent dynamic force between the bubble and the mica is $F(t)=$ $2 \pi \int_{0}^{\infty}[p(r, t)+\Pi(h(r, t))] r d r$. The no-slip or fully immobile boundary condition corresponds to $u=0$ at $z=0$ (mica) and at $z=h$ (bubble) while a full-slip or fully mobile (no tangential stress) condition at the air-water interface corresponds to $\partial u / \partial z=0$ at $z=h$ (bubble). As deformations are small compared to the undeformed

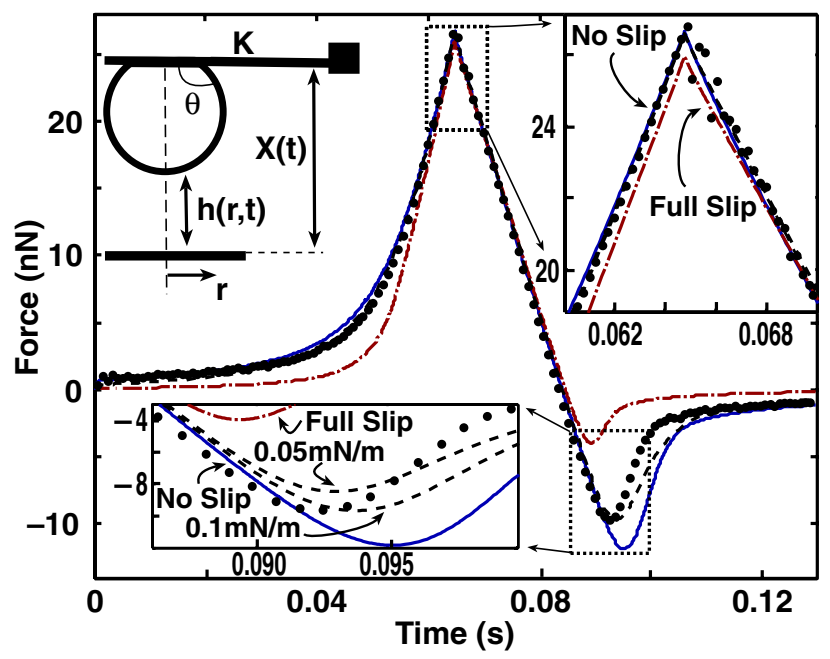

FIG. 2 (color online). Measured dynamic force $F(t)$ between a bubble and a mica plate in $1 \mathrm{mM} \mathrm{NaNO}$ (no SDS) at scan rate $30 \mu \mathrm{m} / \mathrm{s}$ (dotted line). Theoretical results $\left(h_{\text {init }}=1.85 \mu \mathrm{m}\right)$ for the no-slip immobile (solid line, blue), the full-slip fully mobile (dash-dotted line, red) boundary conditions at the bubble and a boundary condition that takes into account the concentration of surface transport of insoluble impurities (dashed line) that lowers the air-water interfacial tension by $\pi_{\text {so }}=0.1 \mathrm{mN} / \mathrm{m}$ or $0.05 \mathrm{mN} / \mathrm{m}$ (inset). 
bubble radius $R_{b}$, the Laplace pressure $\Delta P$ can be approximated by $\left(2 \sigma / R_{b}\right)$ [13] and Eqs. (1) and (2) are to be solved in the inner region $0<r<r_{\max }$. Detailed scaling arguments for the choice of $r_{\max }$ are given elsewhere [13,21], but for a $50 \mu \mathrm{m}$ bubble, $r_{\max } \sim 15 \mu \mathrm{m}$ is sufficient for numerical convergence. The outer boundary condition at $r_{\max }$ that preserves the volume of the bubble and obeys the constraint that the base of the bubble is anchored to the cantilever is [13,21]: $d X / d t=\partial h / \partial t+(\alpha / 2 \pi \sigma) \times$ $(d F / d t) \quad$ at $\quad r=r_{\text {max }}$, where $\alpha=\ln \left(r_{\max } / R_{b}\right)+1+$ $(1 / 2) \ln [1+\cos \theta) /(1-\cos )]-2 \pi \sigma / K$ and $\theta$ is the contact angle of the bubble at the cantilever. The function $X(t)$ is taken from the raw LVDT data and $d X / d t$ is calculated by differentiating a 4th order polynomial fitted to approximately 3000 data points. The initial condition for the film thickness is $h(r, 0)=h_{\text {init }}+r^{2} /\left(2 R_{b}\right)$. The initial distance of closest approach $h_{\text {init }}$ between the bubble and the mica cannot be determined experimentally but can be estimated by fitting to the initial part of the force curve before bubble deformation becomes significant [13].

At surfactant concentrations high enough to ensure a full adsorbed layer at the air-water interface, we obtain excellent agreement between experiment and theory with the no-slip boundary condition at the bubble and mica surfaces (Fig. 1) using parameter values within the range of experimental tolerance. In $1 \mathrm{mM} \mathrm{NaNO}_{3}$, with no SDS, experimental results at a scan speed of $30 \mu \mathrm{m} / \mathrm{s}$ fall between the no-slip (or immobile interface) and the full-slip (or fully mobile interface) boundary conditions (Fig. 2).

To explain the observed forces in electrolyte (no SDS), we propose a surface transport model where a low surface concentration $\Gamma$ of insoluble surface-active impurity is present at the air-water interface which depresses the interfacial tension from the ideal value $\sigma_{0}$ to a lower value: $\sigma=\sigma_{0}-\pi_{s}$, by the surface pressure $\pi_{s}$ of the adsorbed species. Gradients in $\pi_{s}$ along the interface produces the tangential stress boundary condition [32]: $\mu \partial u / \partial z=$ $-d \pi_{s} / d r$ to replace the no-slip boundary condition: $u=0$ at the air-water interface $z=h$. The film thinning equation, Eq. (2), then becomes

$$
\frac{\partial h}{\partial t}=\frac{1}{3 \mu r} \frac{\partial}{\partial r}\left[r h^{3} \frac{\partial p}{\partial r}\right]+\frac{1}{2 \mu r} \frac{\partial}{\partial r}\left[r h^{2} \frac{\partial \pi_{s}}{\partial r}\right] .
$$

The surface-active impurity is assumed to remain at the air-water interface and does not transfer into the aqueous phase during interaction. The two-dimensional ideal gas equation, $\pi_{s}=k T \Gamma(r, t)$ (where $k$ and $T$ are Boltzmann's constant and the absolute temperature), is used to relate the surface pressure and the low surface concentration of impurities. Under these assumptions, the surface convective-diffusion equation that specifies material conservation along the interface is

$$
\frac{\partial \pi_{s}}{\partial t}+\nabla_{t} \cdot\left[\left(u_{s} \hat{r}\right) \pi_{s}\right]=D \nabla_{t}^{2} \pi_{s}
$$

where $D$ is the diffusion coefficient, $\left(u_{s} \hat{\boldsymbol{r}}\right)$ is the tangential velocity at the air-water interface and the operator $\nabla_{t}$ depends only on the radial coordinate $r$ tangential to the interface [33]. From lubrication theory of flow in the thin film, we have $u_{s}=-(h / \mu)\left(\partial \pi_{s} / \partial r\right)-\left(h^{2} / 2 \mu\right)(\partial p / \partial r)$. The relative importance of convective to diffusive effects in Eq. (4) is measured by the Peclet number, $\mathrm{Pe}=\lambda^{2} / D \tau$, where $\lambda$ is a characteristic length in the radial direction, $\tau$ is a characteristic time and $D$ the diffusion coefficient of the surface species of typical magnitude $D \sim 10^{-9} \mathrm{~m}^{2} / \mathrm{s}$. In terms of the capillary number $\mathrm{Ca}=\mu \mathrm{V} / \sigma \sim 10^{-6}$ our governing equations are scaled as follows $[13,21]$ in the radial dimension $r^{*}=R_{b} \mathrm{Ca}^{1 / 4}$ and in time $t^{*}=$ $\mathrm{Ca}^{-1 / 2}\left(R_{b} \mu / \sigma\right)$. The magnitude of the Peclet number $\mathrm{Pe} \sim r^{* 2} / D t^{*} \sim R_{b} V / D \sim 1$ suggests that all three terms in the convective-diffusion equation (4) are of comparable magnitude. We specify the concentration of surface-active impurity by assuming an initial quiescent value for the surface pressure to be $\pi_{s 0}=\sigma_{0}-\sigma(t=$ $0)=0.1 \mathrm{mN} / \mathrm{m}$, which is within uncertainties in determining interfacial tensions. As the experimental time scale is short $(\sim 0.1 \mathrm{~s})$ compared to the diffusion time over the extent of the bubble $\left(\sim R_{b}^{2} / D \sim 2 \mathrm{~s}\right)$, the bubble interfacial tension outside the interaction zone can be assumed to remain at its equilibrium value during the course of the interaction. Thus on the scale of interaction zone: $\pi_{s} \rightarrow$ $\pi_{s 0}$ as $r \rightarrow \infty$. Asymptotic analysis of the film thinning equations then yields the boundary condition: $r\left(\partial \pi_{s} / \partial r\right)+$ $\left(\pi_{s}-\pi_{s 0}\right)=0$ at $r=r_{\max }$ which enables Eqs. (1), (3), and (4) to be solved.

A comparison of this model with experimental forces at various scan rates is given in Fig. 3. This simple model of an isothermal two-dimensional ideal gas encapsulates the physical essence that mobile insoluble surface-active species generate a surface pressure gradient to balance the hydrodynamic viscous tangential stress at the interface. Results for the full-slip (fully mobile) boundary condition can be recovered by setting the quiescent surface pressure, $\pi_{s 0}=0$, while setting $\pi_{s 0} \geq 1 \mathrm{mN} / \mathrm{m}$ will recover results for the no-slip (fully immobile) boundary condition. The sensitivity of the model to $\pi_{s 0}$ in the range $0.05-0.1 \mathrm{mN} / \mathrm{m}$ is illustrated in the insets of Fig. 2.

In conclusion, we have shown experimentally and demonstrated by a detailed model that dynamic forces involving bubbles in the absence of added surfactant are very sensitive to small amounts of surface-active impurity. The surface concentrations and resultant surface pressures are sufficiently low to be within experimental tolerance of surface tension measurements and the effects of spatial variations in the interfacial tension in the Young-Laplace equation are negligible. While the Navier slip model can be made to fit the measured dynamic force with a post hoc slip length of $17-23 \mathrm{~nm}$, our surface transport model provides a semiquantitative physical mechanism that underpins this observation. 


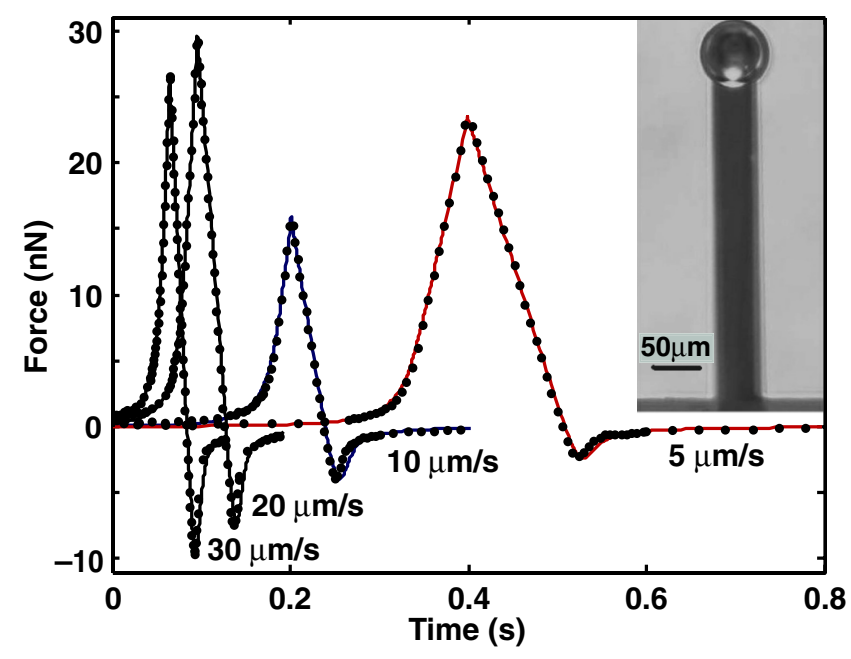

FIG. 3 (color online). Time variations of the measured dynamic force $F(t)$ between a bubble and a mica plate in $1 \mathrm{mM}$ $\mathrm{NaNO}_{3}$ (no SDS) (dotted line) compared to the surface transport model (solid line) with $\pi_{s 0}=0.1 \mathrm{mN} / \mathrm{m}$ at initial separations $h_{\text {init }}: 1.96 \mu \mathrm{m}(5 \mu \mathrm{m} / \mathrm{s}), 2.11 \mu \mathrm{m} \quad(10 \mu \mathrm{m} / \mathrm{s}), \quad 1.76 \mu \mathrm{m}$ $(20 \mu \mathrm{m} / \mathrm{s})$ and $1.85 \mu \mathrm{m}(30 \mu \mathrm{m} / \mathrm{s})$. Model parameters with experimental values in brackets are: mica surface potential: $-90 \mathrm{mV}(-95 \pm 10 \mathrm{mV}$ [34]), bubble surface potential: $-35 \mathrm{mV} \quad(-30 \pm 10 \mathrm{mV}$ [36]), interfacial tension $\sigma$ : $73 \mathrm{mN} / \mathrm{m}(72 \pm 1 \mathrm{mN} / \mathrm{m})$, bubble radius $R_{b}: 56 \mu \mathrm{m}(55 \pm$ $2 \mu \mathrm{m})$ and contact angle $\theta: 152^{\circ}\left(150 \pm 5^{\circ}\right)$. Inset: Photograph of the bubble on the rectangular cantilever.

This work is supported in part by the Australian Research Council through funding of the Particulate Fluids Processing Centre and the Australian Minerals Science Research Institute jointly with AMIRA International and the State Governments of Victoria and South Australia. O.M. is supported by the University of Melbourne.

*rrd@unimelb.edu.au

†D.Chan@unimelb.edu.au

[1] G. M. Whitesides, Nature (London) 442, 368 (2006).

[2] C. A. Taylor and M. T. Draney, Annu. Rev. Fluid Mech. 36, 197 (2004).

[3] D. Lohse, Phys. Today 56, No. 2, 36 (2003).

[4] D. Y.C. Chan and R. G. Horn, J. Chem. Phys. 83, 5311 (1985).

[5] C. Cottin-Bizonne, B. Cross, A. Steinberger, and E. Charlaix, Phys. Rev. Lett. 94, 056102 (2005).

[6] C. Cottin-Bizonne, S. Jurine, and J. Baudry et al., Eur. Phys. J. E 9, 47 (2002).

[7] R. G. Horn, D. T. Smith, and W. Haller, Chem. Phys. Lett. 162, 404 (1989).
[8] J. N. Israelachvili, J. Colloid Interface Sci. 110, 263 (1986).

[9] J. N. Connor and R. G. Horn, Faraday Discuss. 123, 193 (2003).

[10] L. Y. Clasohm, J. N. Connor, and O. I. Vinogradova et al., Langmuir 21, 8243 (2005).

[11] R. Manica, J. N. Connor, and S. L. Carnie et al., Langmuir 23, 626 (2007).

[12] R. Manica, J. N. Connor, and L. Y. Clasohm et al., Langmuir 24, 1381 (2008).

[13] R. Manica, J. N. Connor, and R. R. Dagastine et al., Phys. Fluids 20, 032101 (2008).

[14] C. Cottin-Bizonne, A. Steinberger, and B. Cross et al., Langmuir 24, 1165 (2008).

[15] E. Bonaccurso, H.-J. Butt, and V.S. J. Craig, Phys. Rev. Lett. 90, 144501 (2003).

[16] E. Bonaccurso, M. Kappl, and H.-J. Butt, Phys. Rev. Lett. 88, 076103 (2002).

[17] C. Neto, V. S. J. Craig, and D. R. M. Williams, Eur. Phys. J. E 12, S71 (2003).

[18] C. Neto, D. R. Evans, and E. Bonaccurso et al., Rep. Prog. Phys. 68, 2859 (2005).

[19] R. Pit, H. Hervet, and L. Leger, Phys. Rev. Lett. 85, 980 (2000).

[20] C. D. F. Honig and W. A. Ducker, Phys. Rev. Lett. 98, 028305 (2007).

[21] S. L. Carnie, D. Y. C. Chan, and C. Lewis et al., Langmuir 21, 2912 (2005).

[22] R. R. Dagastine, T. T. Chau, and D. Y.C. Chan et al., Faraday Discuss. 129, 111 (2005).

[23] R. R. Dagastine, R. Manica, and S.L. Carnie et al., Science 313, 210 (2006).

[24] R. R. Dagastine, G. W. Stevens, and D. Y. C. Chan et al., J. Colloid Interface Sci. 273, 339 (2004).

[25] G. B. Webber, S. A. Edwards, and G. W. Stevens et al., Soft Matter 4, 1270 (2008).

[26] G. B. Webber, R. Manica, and S. A. Edwards et al., J. Phys. Chem. C 112, 567 (2008).

[27] A. Steinberger, C. Cottin-Bizonne, P. Kleimann, and E. Charlaix, Phys. Rev. Lett. 100, 134501 (2008).

[28] R. Manica, E. Klaseboer, and D. Y. C. Chan, Soft Matter (to be published).

[29] I. U. Vakarelski, J. Lee, and R. R. Dagastine et al., Langmuir 24, 603 (2008).

[30] J. P. Cleveland, S. Manne, and D. Bocek et al., Rev. Sci. Instrum. 64, 403 (1993).

[31] D. McCormack, S. L. Carnie, and D. Y. C. Chan, J. Colloid Interface Sci. 169, 177 (1995).

[32] V. G. Levich, Physicochemical Hydrodynamics (Prentice Hall, Englewood Cliffs, New Jersey, 1962).

[33] A. K. Chesters and I. B. Bazhlekov, J. Colloid Interface Sci. 230, 229 (2000).

[34] P. J. Scales, F. Grieser, and T. W. Healy, Langmuir 6, 582 (1990).

[35] J. Y. Kim and M. G. Song, J. Colloid Interface Sci. 223, 285 (2000).

[36] M. Takahashi, J. Phys. Chem. B 109, 21858 (2005). 